\title{
Comparison of unitary exocytic events in pituitary lactotrophs and in astrocytes: modeling the discrete open fusion-pore states
}

\author{
Doron Kabaso ${ }^{1,2}$, Jernej Jorgačevski ${ }^{2,3}$, Ana I. Calejo ${ }^{2,4}$, Ajda Flašker ${ }^{2}$, Alenka Guček², Marko Kreft ${ }^{2,3,5}$ \\ and Robert Zorec ${ }^{2,3 *}$ \\ ${ }^{1}$ Laboratory of Biophysics, Faculty of Electrical Engineering, University of Ljubljana, Ljubljana, Slovenia \\ ${ }^{2}$ Laboratory of Neuroendocrinology-Molecular Cell Physiology, Faculty of Medicine, Institute of Pathophysiology, University of Ljubljana, Ljubljana, Slovenia \\ ${ }^{3}$ Celica Biomedical Center, Ljubljana, Slovenia \\ ${ }^{4}$ Departamento de Biologia e CESAM, Universidade de Aveiro, Aveiro, Portugal \\ ${ }^{5}$ Department of Biology, Biotechnical Faculty, University of Ljubljana, Ljubljana, Slovenia
}

\section{Edited by:}

Keith Murai, McGill University,

Canada

Reviewed by:

Vladimir Parpura, University

of Alabama, USA

Gerhard Thiel, TU-Darmstadt,

Germany

*Correspondence:

Robert Zorec, Laboratory of Neuroendocrinology-Molecular Cell

Physiology, Medical Faculty,

University of Ljubljana, Zaloška

cesta 4, SI-1000 Ljubljana, Slovenia.

e-mail: robert.zorec@mf.uni-lj.si
In regulated exocytosis the merger between the vesicle and the plasma membranes leads to the formation of an aqueous channel (a fusion-pore), through which vesicular secretions exit into the extracellular space. A fusion pore was thought to be a short-lived intermediate preceding full-fusion of the vesicle and the plasma membranes (full-fusion exocytosis). However, transient exocytic events were also observed, where the fusion-pore opens and closes, repetitively. Here we asked whether there are different discrete states of the open fusion-pore. Unitary exocytic events were recorded by the high-resolution cell-attached patch-clamp method in pituitary lactotrophs and brain astrocytes. We monitored reversible unitary exocytic events, characterized by an on-step, which is followed by an off-step in membrane capacitance $\left(C_{m}\right)$, a parameter linearly related to the membrane area. The results revealed three categories of reversible exocytic events (transient fusion-pore openings), which do not end with the complete integration of the vesicle membrane into the plasma membrane. These were categorized according to the observed differences in the amplitude and sign of the change in the real $(R e)$ parts of the admittance signals: in case I events $(R e \approx 0)$ fusion pores are relatively wide; in case II (Re $>0)$ and case III $(R e<0)$ events fusion pores are relatively narrow. We show that case III events are more likely to occur for small vesicles, whereas, case II events are more likely to occur for larger vesicles. Case III events were considerably more frequent in astrocytes than in lactotrophs.

Keywords: capacitance measurements, equivalent circuit, transient fusion-pore, modeling, astrocytes

\section{INTRODUCTION}

In regulated exocytosis the fusion between the vesicle and the plasma membrane is important not only for the secretion of signaling molecules, such as hormones and transmitters, but is also key for the membrane recycling and for the translocation of membrane receptors and other proteins to the plasma membrane (White, 1992; Jahn et al., 2003; Vardjan et al., 2009). The membrane fusion process is an energetically unfavorable event, since it consists of bringing close to each other negatively charged opposing membranes, and involves the bending of a lipid-bilayer into a highly curved structure, which is assembled by different proteins and lipids (Kozlov and Markin, 1983). Following the merger of the two membranes, an important step in regulated exocytosis is the formation of a fusion-pore, an aqueous channel connecting the vesicle lumen and the exterior of the cell (reviewed in Chernomordik and Kozlov, 2008).

The nature of this structure can be studied by the electrophysiological patch-clamp membrane capacitance $\left(C_{m}\right)$ technique, which allows monitoring interactions of a single vesicle with the plasma membrane (Neher and Marty, 1982; Lindau and Neher,
1988; Lollike et al., 1995), and the dynamics of an individual fusion-pore. In addition to simple discrete step increases in $C_{m}$, which were considered to represent full-fusion exocytic events (Neher and Marty, 1982), some of the unitary exocytic events are transient in nature, characterized by repetitive discrete on-steps (increases) followed by an equal amplitude off-steps (decreases) in $C_{m}$. The increment and the ensuing decrement step in $C_{m}$ are considered to be due to a transient fusion-pore opening of a fused vesicle (Heuser and Reese, 1973; Alvarez de Toledo et al., 1993; Vardjan et al., 2007). These events were also termed transient or reversible exocytic events, also $\gg$ kiss-and-run $\ll$ exocytosis. Transient fusion-pore openings are considered physiologically relevant, since the fusion-pore is not formed upon each round of exocytosis (Ceccarelli et al., 1972; Kozlov and Markin, 1983). Moreover, a narrow fusion-pore, once it is established, appears to be energetically relatively favorable (Jorgačevski et al., 2010, 2011).

These conclusions were made on the basis of monitoring repetitive unitary exocytic events in which the narrowness of the fusion-pore can be determined from optical 
and electrophysiological measurements (Vardjan et al., 2007; Jorgačevski et al., 2008). In electrophysiological measurements specifically, by determining fusion-pore conductance $\left(G_{p}\right)$ and vesicle capacitance $\left(C_{v}\right)$ from admittance measurements (Lindau, 1991; Lollike and Lindau, 1999). In these recordings, changes observed in the imaginary $(\Delta I m)$ and in the real $(\Delta R e)$ parts of admittance signals reflect changes in $C_{v}$ and $G_{p}$, which can be used to determine vesicle diameter and fusion pore diameter (Lindau, 1991; Rosenboom and Lindau, 1994). Occasionally, the admittance traces associated with a transient fusion-pore opening exhibit an incremental or a decremental cross-talk in the Re signal (Breckenridge and Almers, 1987; Lindau, 1991; Henkel et al., 2000). However, the underlying mechanisms responsible for these non-zero projections in the Re signal are not fully understood.

In previous studies, the equivalent circuit of the fusion pore was reported (Lindau and Neher, 1988; Scepek and Lindau, 1993; Lollike et al., 1995). It was shown analytically that the incremental cross-talk projection on the Re signal could be due to the fusionpore opening devoid of complete vesicle membrane integration into the plasma membrane (Lindau, 1991; Henkel et al., 2000). By studying pituitary lactotrophs, an ideal cell preparation to study secretory activity at the single vesicle level (Stenovec et al., 2004; Vardjan et al., 2007), and astrocytes which release gliotransmitters (Parpura et al., 1994) by likely employing regulated exocytosis (Parpura and Zorec, 2010), we compared the properties of unitary exocytic events. Using equivalent circuit analysis, we here demonstrate that the decremental cross-talk projection on the $R e$ signal depends on the $G_{p}$ as well as on the size of the fused vesicle. Moreover, these results indicate the existence of a very narrow, nearly closed, open fusion-pore state in pituitary lactotrophs and in astrocytes.

\section{MATERIALS AND METHODS CELL CULTURES}

Primary lactotroph and astrocyte cultures were prepared from adult male (lactotrophs) and 2-3 days old female Wistar rats as described previously (Schwartz and Wilson, 1992; Ben-Tabou et al., 1994; Jorgačevski et al., 2008). After the isolation we plated cells on poly-L-lysine-coated coverslips and maintained them in high-glucose DMEM (Invitrogen) medium, supplemented with $10 \%$ newborn calf serum and $2 \mathrm{mM}$ L-glutamine in an atmosphere of humidified air (95\%) and $\mathrm{CO}_{2}(5 \%)$. We cared for the experimental animals in accordance with the International Guiding Principles for Biomedical Research Involving animals, developed by the Council for International Organizations of Medical Sciences, and the Directive on Conditions for Issue of License for Animal Experiments for Scientific Research Purposes (Official Gazette of the Republic of Slovenia 40/85 and 22/87). The procedures using animals were approved by the Veterinary Administration of the Republic of Slovenia (approval no. 3440129/2009/2).

\section{ELECTROPHYSIOLOGY}

Cell-attached capacitance measurements on isolated rat lactotrophs and astrocytes were performed with a dual-phase lock-in patch-clamp amplifier (SWAM IIC and SWAM CELL, Celica, Ljubljana, Slovenia) as described (Kreft and Zorec, 1997; Vardjan et al., 2007; Jorgačevski et al., 2010). Briefly, a sine wave voltage $(1591$ or $6400 \mathrm{~Hz}, 111 \mathrm{mV})$ was applied to the pipette, while holding the pipette potential at $0 \mathrm{mV}$. The phase of the lock-in amplifier was adjusted to nullify the changes in Re. A $10 \mathrm{fF}$ calibration pulse was manually generated every $10 \mathrm{~s}$ to ensure correct phase angle settings. We used thick-walled, fire polished glass pipettes, which were heavily coated with a resin (Sylgard ${ }^{\circledR} 184$ ) and had a resistance of $2-5 \mathrm{M} \Omega$.

\section{THE EQUIVALENT CIRCUIT OF A TRANSIENT FUSION PORE}

A patch-clamp configuration could be approximated by the series combination of the membrane and the access resistance $\left(R_{A}\right)$ of the pipette tip through the patch (Figure 1A). The membrane included a parallel setup of the whole-cell $C_{m}$ and the membrane conductance $\left(G_{M}\right)$. When a small vesicle fused with the patch region, the patch-clamp system could detect the fusion event by the observed changes in the admittance measurement. It has been previously confirmed that the fusion of a vesicle was accompanied by an increase in the measured $C_{m}$ (Neher and Marty, 1982). The equivalent circuit of the patch-configuration with a fused vesicle is shown in Figure 1B. The vesicle $C_{m}$ is denoted by $C_{v}$, and the fusion-pore conductance is denoted by $G_{p}$.

In the present analysis, all the parameters except $G_{p}$ were held constant. It was assumed that $C_{v}<<C_{m}$ or $\omega C_{v}<<1 / R_{A}$, which are reasonable assumptions, since the vesicle $C_{m}$ (vesicle surface area) is considerably smaller than the cell $C_{m}$ (cell surface area), and the patch resistance is considerably greater than the capacitor load of the vesicle $\left(\omega C_{v}\right)$. The admittance change $(\Delta Y)$ describes the change in the admittance between the open and nearly closed state of the fusion pore. According to the equivalent circuit, the admittance difference is (Lindau, 1991):

$$
\Delta Y=T^{2}(\omega)\left(\frac{\left(\omega C_{v}\right)^{2} / G_{p}}{1+\left(\omega C_{v} / G_{p}\right)^{2}}+i \frac{\omega C_{v}}{1+\left(\omega C_{v} / G_{p}\right)^{2}}\right)
$$

where $T^{2}(\omega)$ stands for the factor $T^{2}(\omega)=1 /\left(1+R_{A} G_{M}+\right.$ $\left.i \omega C_{m} R_{A}\right)^{2}=|T(\omega)|^{2} \cdot e^{i \theta}$, and $i$ is $\sqrt{-1}$. The admittance change

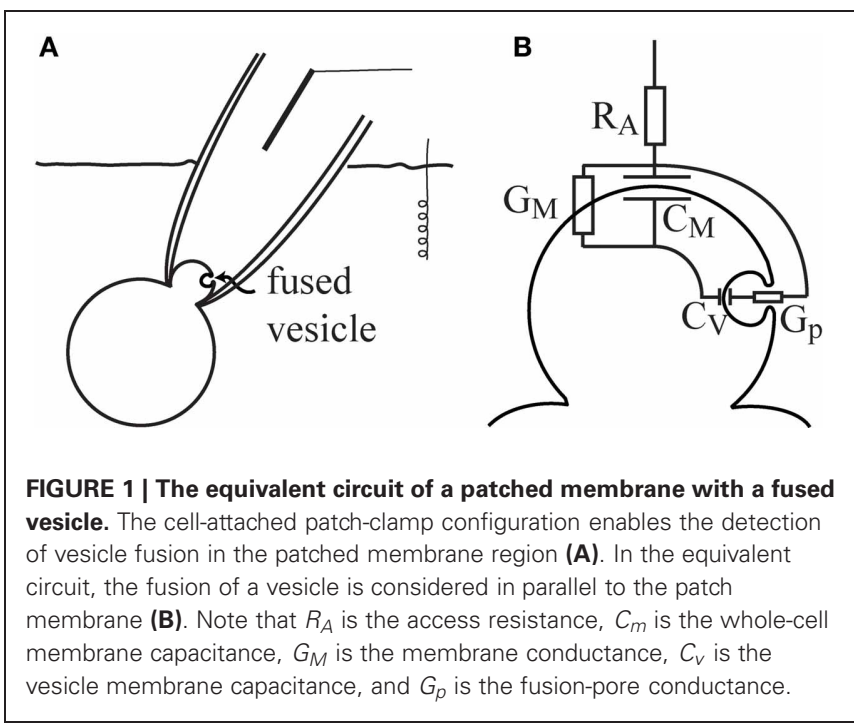


$(\Delta Y)$ is an imaginary number, in which the first term in the parenthesis is the $\Delta R e$, and the second term in the parenthesis is the $\Delta I m$. For the sake of simplicity, the fusion pore state is denoted as fully open or incompletely open. In the fully open state, the $G_{p}$ is infinite $\left(G_{p} \rightarrow \infty\right)$, and the $\Delta R e$ in Equation (1) vanishes, which gives $\Delta Y=i T^{2}(\omega) \omega C_{v}$. On the other hand, when there is incomplete fusion, the $G_{p}$ can be on the same scale as $\omega C_{v}$. As a result, both the $\Delta R e$ and $\Delta I m$ have a finite value, which can be used for the calculation of the unknown $G_{p}$ (Breckenridge and Almers, 1987). The $C_{v}$ and the $G_{p}$ can be obtained from the real and imaginary parts (Lindau, 1991; Lollike and Lindau, 1999), as follows:

$$
\begin{aligned}
C_{v} & =\frac{\Delta R e^{2}+\Delta I m^{2}}{\Delta I m} / \omega \\
G_{p} & =\frac{\Delta R e^{2}+\Delta I m^{2}}{\Delta R e} .
\end{aligned}
$$

\section{RESULTS}

\section{ADMITTANCE MEASUREMENTS OF THE THREE CASES OF TRANSIENT EXOCYTIC EVENTS}

The admittance measurements of repetitive opening and closure of fusion-pores were obtained in lactotrophs (Figures 2Ai,ii) and in astrocytes (Figures $\mathbf{2 B i i i , i v ) , ~ w h e r e ~ r e p r e s e n t a t i v e ~ t r a n - ~}$ sient exocytic events are shown, denoted as cases I, II, and III on Figure 2. The asterisks in these recordings indicate calibration pulses which were used to adjust the phase of the lock-in amplifier. The events in panels Figures $\mathbf{2 A , B}$ were recorded in the same membrane patch of a lactotroph and an astrocyte, respectively. Using Equation (2), the corresponding vesicle surface area in $\mathrm{fF}$ is calculated by incorporating $\Delta I m$ and $\Delta R e$ estimated for each event from the admittance records. On Figure 2A (recorded in lactotrophs) the $C_{v}$ in case I, case II, and case III, was 0.9, 4, and $1.2 \mathrm{fF}$, respectively. Whereas, on Figure 2B, where representative recordings in astrocytes are shown, the $C_{v}$ was in case I $(0.3 \mathrm{fF})$, case II $(1.6 \mathrm{fF})$, and case III $(0.6 \mathrm{fF})$. One can note that the amplitude in $I m$, reflecting $C_{v}$, appears larger in events of case II in comparison with case I events. Case I events exhibit an incremental change in $I m$ trace and are devoid of projection on the $R e$ trace. In case II events and increment in Im trace is associated with an incremental projection on the Re trace, whereas, in case III events an increment in Im trace is associated with a decremental projection on the $R e$ trace. In Figure $2 \mathrm{C}$ the three cases of transient exocytic events are diagrammatically presented as pre-fused vesicles with an initial narrow fusion-pore (narrower than the detection limit of the recording system), which can reversibly widen to a larger diameter - a state that can be detected electrophysiologically.

The experimental datasets used in our analysis, include $\Delta R e$ and $\Delta I m$ of cases I-III obtained in lactotrophs (Figures 3A-D) and astrocytes (Figures $\mathbf{3 E}-\mathbf{H}$ ). The $G_{p}$ of each event is calculated from $\Delta R e$ and $\Delta I m$ of cases II ( $\Delta R e$ incremental) and III $(\Delta R e$ decremental). Figures $\mathbf{3 A}-\mathbf{H}$ shows scatter plots of $\Delta R e$ and $\Delta I m$ as a function of Gp. The relationships between $\Delta I m$ versus $\Delta R e$ are plotted for the datasets of incremental and decremental $\Delta R e$ projections. A strong linear relationship $(r=0.79$ in Figure 3C; $r=0.75$ in Figure 3G) was revealed between $\Delta I m$ and $\Delta R e$ in

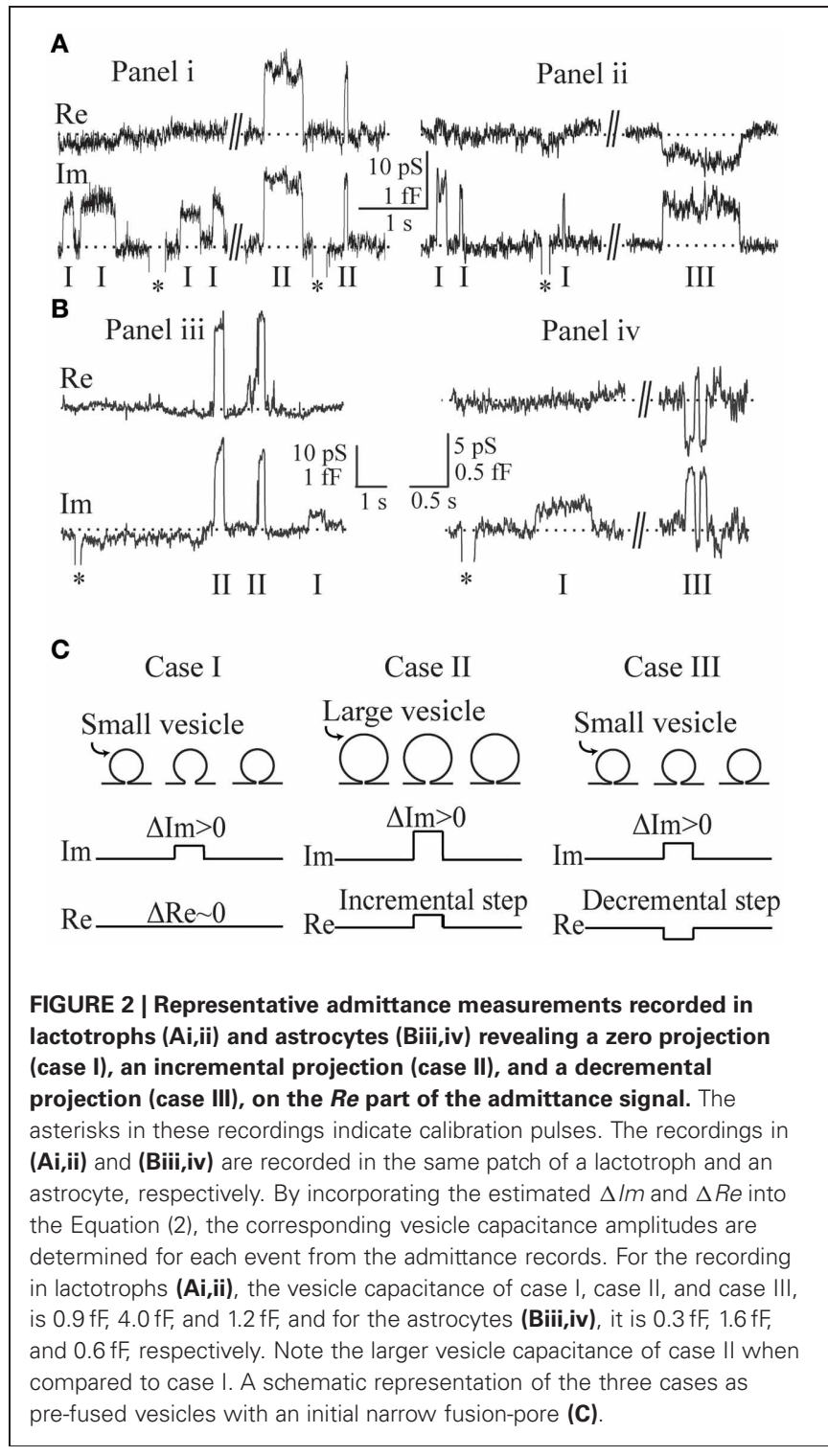

the incremental $\Delta R e$ projection group (Figures 3C,G). The slope of this relationship in astrocytes is smaller than one (Figure $\mathbf{3 G}$ ), however that in lactotrophs is close to unity (Figure 3C), suggesting that $\Delta R e$ and $\Delta I m$ are of similar size. The non-zero $\Delta R e$ can be attributed to the incomplete opening of the fusion-pore, which is accompanied by the increased fusion-pore resistance, whereas, the fully open fusion-pore exhibit a negligible resistance. On the other hand, in the decremental $\Delta R e$ projection datasets, a weak correlation between $\Delta I m$ and $\Delta R e$ was revealed $(r=0.49$ in Figure 3D; $r=0.08$ in Figure 3H). We noted that the datasets of decremental $R e$ projections are clustered more in the range of small $G_{p}$ (Figures 3B,F).

\section{REPRODUCING THE THREE CASES OF ADMITTANCE MEASUREMENTS}

Next, we considered the mechanisms responsible for the incremental and decremental $\Delta R e$ projections in the admittance measurements of the two cell types. According to the equivalent electrical circuit, the change in the real $(\Delta R e)$ and imaginary 

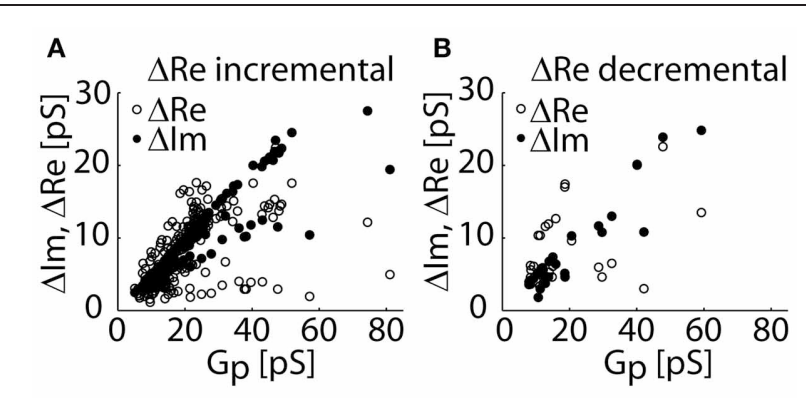

C

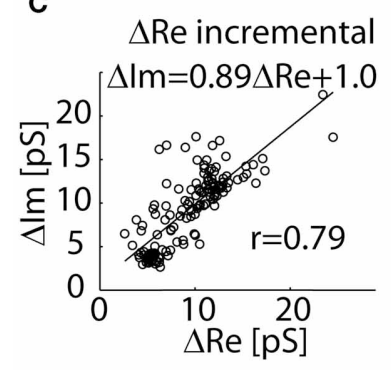

D
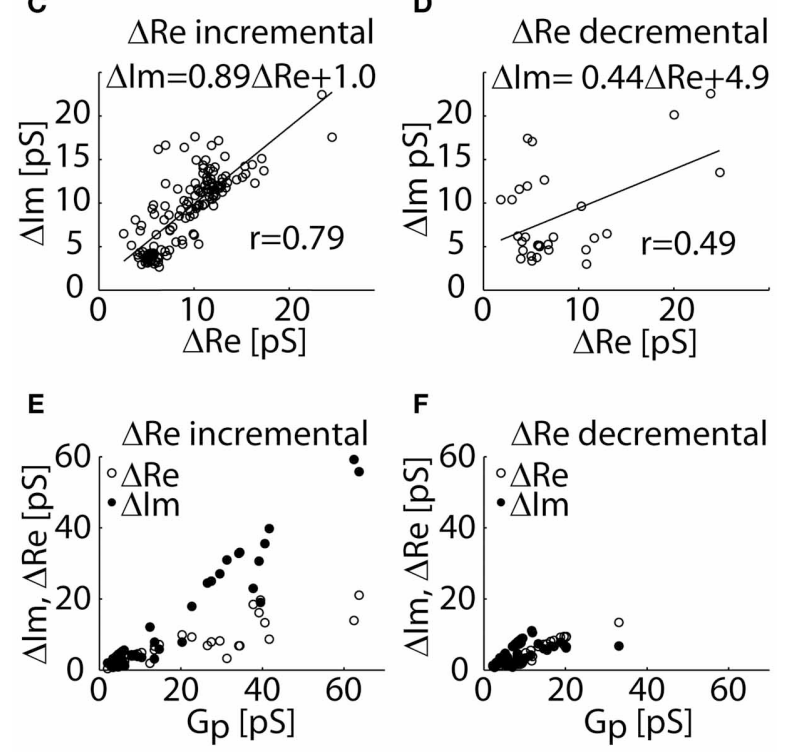

G

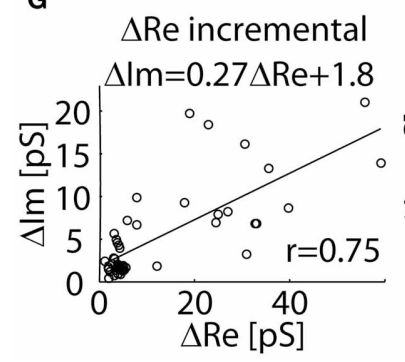

H

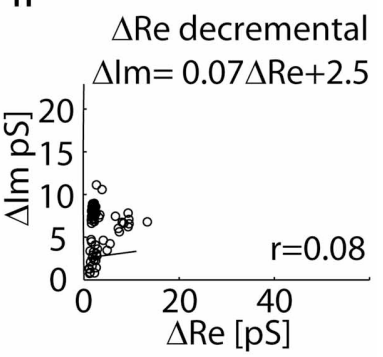

FIGURE 3 | The experimental datasets of $\Delta R e$ and $\Delta / m$ of admittance measurements recorded in lactotrophs (A-D) and astrocytes (E-H) incremental (case II) and decremental (case III) $\Delta R e$ projections. The distributions of $\Delta R e$ and $\Delta / m$ as a function of $G_{p}$ for the datasets of incremental $\Delta R e(\mathbf{A}, \mathbf{E})$ and for the decremental $\Delta R e(\mathbf{B}, \mathbf{F})$ datasets. A scatter plot of $\Delta / m$ against $\Delta R e$ reveals a strong linear relationship in the incremental $\Delta R e$ datasets of projections (C,E), and a weak linear correlation in the dataset of decremental $\Delta R e$ projections $(\mathbf{D}, \mathbf{H})$.

$(\Delta I m)$ parts of admittance signals can be employed for the calculation of $C_{v}$ and $G_{p}$ (Lindau, 1991; Lollike and Lindau, 1999).

In Figure 4, $\Delta R e$ and $\Delta I m$ are plotted as a function of $G_{p}$ calculated for intermediate $\left(C_{v}=1.2 \mathrm{fF}\right)$, large $\left(C_{v}=4 \mathrm{fF}\right)$, and small $\left(C_{v}=0.5 \mathrm{fF}\right)$ vesicles, representing events of case I, II, and III, respectively. The fact that in case I there is no projection on the Re trace can be reproduced by a change from a fully closed fusion-pore state $\left(G_{p}=0 \mathrm{pS}\right)$ to a fully open fusion-pore state
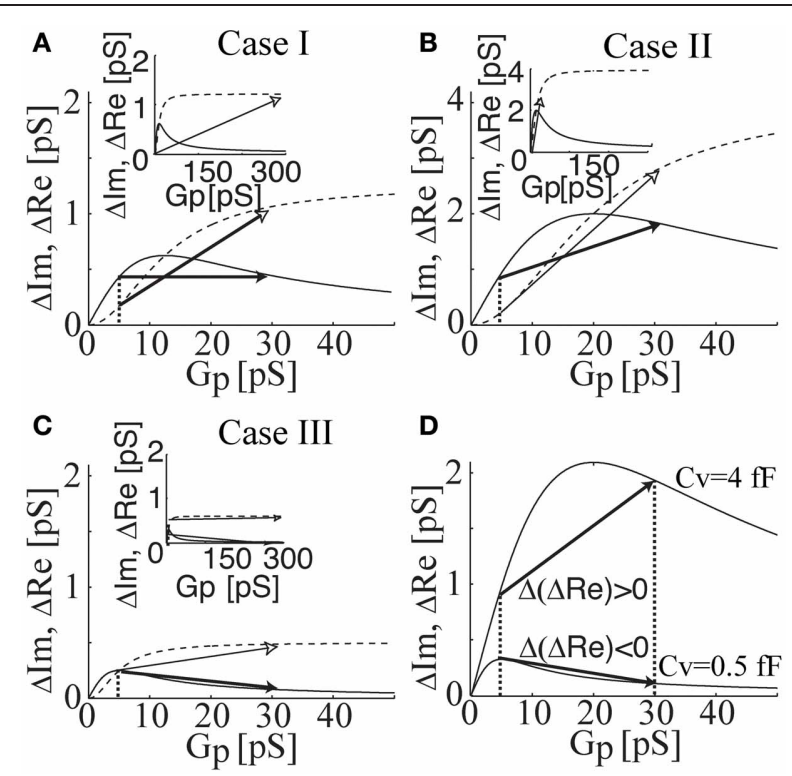

FIGURE 4 | The relationships of $\Delta R e$ (solid lines) and $\Delta / m$ (dahsed lines) as a function of $G_{p}$ calculated for intermediate $\left(C_{v}=1.2 \mathrm{fF}\right.$; case I), large $\left(C_{v}=4 \mathrm{fF}\right.$; case II), and small $\left(C_{v}=0.5 \mathrm{fF}\right.$; case III) vesicle size. Note that the change in $\Delta R e$ and $\Delta / m$ are drawn by a full and an open arrow, respectively. The zero projection on the Re part is reproduced by a change from a fully closed state to a fully open fusion-pore state (see inset) or by a change from a nearly closed state to an incompletely open fusion-pore state (A). The incremental projection in case II is reproduced by a change from nearly closed to incompletely open state (B). The decremental projection in case III can be reproduced, only when the pre-fused state is nearly closed (C). In addition, the change in $\Delta R e$ can be considerably larger than the change in $\Delta / \mathrm{m}$ (see inset). Overlay of $\Delta R e(G p)$ of cases I and III reveals the effect of vesicle size on the projection type (D). By having the same pre-fused state (i.e., the same fusion pore conductance), the incomplete fusion-pore opening of a large vesicle size leads to an incremental projection, whereas, the same incomplete opening of a small vesicle causes a decremental projection in $R e$.

(e.g., $G_{p}>500 \mathrm{pS}$ in lactotrophs and $G_{p}>70 \mathrm{pS}$ in astrocytes) or by a change from a nearly closed fusion-pore state (e.g., $G_{p}=5 \mathrm{pS}$ in lactotrophs and $G_{p}=2 \mathrm{pS}$ in astrocytes) to an incompletely open fusion-pore state (e.g., $G_{p}=30 \mathrm{pS}$ ) (Figure 4A). The positive projection in case II can be due to fusion-pore opening from a fully closed state, or a nearly closed state, to an incompletely open state (Figure 4B). On the other hand, the negative projection in case III can be reproduced only when the pre-fused state is nearly closed (Figure 4C), which suggests the possible existence of a nearly closed state of the fusion-pore. The underlying hypothesis for the different transient exocytic event cases is that the incremental and decremental projections to the Re trace are due to differences in the fused vesicle size and its nearly closed fusionpore state. According to the presented equivalent circuit, small vesicles will tend to exhibit decremental $\Delta R e$ projections when their initial fused state is nearly closed. In Figure $4 \mathrm{D}$, the relationships of $\Delta R e$ as a function of $G_{p}$ of a small and a large vesicle are demonstrated. The overlay of the two relationships reveals that the maximum of $\Delta R e$ is at a lower $G_{p}\left(G_{p}=5 \mathrm{pS}\right)$ for the small vesicle than the counterpart $\left(G_{p}=20 \mathrm{pS}\right)$ in the case of the large vesicle. Assuming that initial vesicle fusion status arises from the 
same nearly closed state (e.g., $G_{p}=5 \mathrm{pS}$ ), the incomplete opening of the fusion pore would lead to a positive projection in the case of the large vesicle and a decremental projection in the $\Delta R e$ in the case of the small vesicle (Figure 4D).

\section{DISCUSSION}

In the present paper, we analyzed the discrete open fusion-pore states as well as the conditions under which these states existed. Three different cases are categorized, in which the transitions between discrete states of the fusion-pore do not end with the complete fusion (i.e., exocytosis) of a vesicle and the plasma membrane.

These cases are evident from the changes in the real $(\Delta R e)$ and imaginary $(\Delta I m)$ parts of admittance measurements (Figure 2). In the first case (denoted as case I), the event is characterized by a step increase in $\Delta I m$ and an approximately zero $\Delta R e$. In the second case (denoted as case II), both $\Delta I m$ and $\Delta R e$ are exhibiting a step increase. The third case (denoted as case III) has an incremental $\Delta I m$ and a decremental $\Delta R e$. The underlying assumption of our model is that the non-zero $\Delta R e$ is due to an incomplete vesicle fusion-pore opening, and that the initial status of the fused vesicle may exhibit a non-zero $G_{p}$ (Figure 2). The equivalent circuit of a patch-clamp configuration was constructed, in which the fused vesicle is considered in parallel to the cell plasma membrane (Figure 1). The fused vesicle is assumed to be connected to the cell membrane via an aqueous channel (the fusion-pore). The $C_{v}$ and the $G_{p}$ are derived from the imaginary $(\Delta I m)$ and real $(\Delta R e)$ parts of the admittance measurements. The experimental data of $\Delta R e$ and $\Delta I m$ are obtained from admittance measurements in lactotrophs and astrocytes (Figure 2). In case II, a strong linear relationship between $\Delta R e$ and $\Delta I m$ suggests that the fusion-pore opening of a vesicle is incomplete (Figures $\mathbf{3 C , G}$ ). In case III, there is a weak correlation between $\Delta R e$ and $\Delta I m$ (Figures 3D,H). According to the relationships of $\Delta R e$ and $\Delta I m$ as a function of $G_{p}$, it is demonstrated that the decremental $R e$ projection is more likely to occur for small vesicles (Figure 4C). Finally, the present calculations reveal that while the incomplete opening of the fusion-pore may be accompanied by the same change in fusion-pore conductance, the resulted projection is predicted to be decremental for the relatively small vesicle and incremental for the large vesicle (Figure 4D).

The high bending energy during the formation of a fusionpore can be overcome by the assembly of curvature membrane constituents (proteins and lipids) (Kozlov and Markin, 1983; Jorgačevski et al., 2010; Kabaso et al., 2012; Jesenek et al., 2012). The stability of the fusion-pore of a fused vesicle can be due to anionic lipids of negative spontaneous curvature, modulating the anterior pituitary cells. J. Physiol.

Alvarez de Toledo, G., Fernandez-

Chacon, R., and Fernandez, J.

(1993). Release of secretory products during transient vesicle fusion. Nature 363, 554-558.

Ben-Tabou, S., Keller, E., and Nussinovitch, I. (1994). Mechanosensitivity of voltagegated calcium currents in rat

formation of the fusion pore (Coorssen and Rand, 1990; Chen and Rand, 1997; Churchward et al., 2008; Rituper et al., 2012). It is then possible that the density of these curvature membrane constituents can affect the $G_{p}$ of the nearly closed fusion-pore state as well as the projection type and amplitude. The possible incomplete fusion-pore state opens a communication venue, in which the passage of small molecules such as ions may be facilitated continuously through the narrow pore. However, larger molecular weight molecules are unable to exit the narrow fusion pore.

What appears interesting is that in both cell types, electrically excitable (pituitary lactotrophs) and electrically non-excitable (brain astrocytes), fusion-pore properties appear to be shared. While vesicles in the lactotrophs exhibit larger diameters and are therefore more accessible to experimentation (Stenovec et al., 2004; Vardjan et al., 2007), secretory vesicles in astrocytes appear to exhibit relatively large and relatively small diameters. The latter ones can be revealed by the higher-resolution cell-attached patchclamp measurements (Kreft and Zorec, 1997). Interestingly, it is the smaller ones that exhibit fusion-pores with extremely narrow fusion-pore diameters (Figure 4). The probability of observing an increment in Im trace, associated with a decremental change in $R e$ trace, indicates that a relatively large fraction of vesicles, which are already fused with the plasma membrane, exhibit a very narrow fusion pore. These may pass protons as has been reported previously by using a $\mathrm{pH}$-sensitive vesicle luminal fluorophore in lactotrophs (Vardjan et al., 2007) and in astrocytes (Malarkey and Parpura, 2011). However the relatively large abundance of these events recorded in astrocytes, may not mean that fusionpore openings mediate a productive release of gliotransmitters. A $G_{p}$ of less than $5 \mathrm{pS}$ means that the fusion pore diameter is less than $0.2 \mathrm{~nm}$, too narrow to pass even glutamate or acetylcholine (Vardjan et al., 2007). These results are consistent with the view that fusion-pores, when they are established, are relatively stable structures (Jorgačevski et al., 2010). The regulation of exocytotic release of hormones and transmitters, thus involves also the regulation at the fusion-pore level, at the level, when the fusion-pore has been already established, but is too narrow to functionally contribute to the exit of secretions form the vesicle lumen. In the present paper we have revealed that fusion-pores may exhibit distinct fusion-pore diameters and the future work will have to address question of how these open fusion-states transit to a release productive state.

\section{ACKNOWLEDGMENTS}

This work was supported by grants P3 03100381 and J33632-1683-10 from the Ministry of Higher Education, Sciences and Technology of the Republic of Slovenia. 476, 29-39.

junctions by prolonged tetanic stimulation. J. Cell Biol. 54, 30-38.

L. J., and Almers, W. (1987). Currents through the fusion pore that forms during exocytosis of a secretory vesicle. Nature 328, 814-817.

Ceccarelli, B., Hurlbut, W. P., and Mauro, A. (1972). Depletion of vesicles from frog neuromuscular
Chen, Z., and Rand, R. P. (1997). The influence of cholesterol on phospholipid membrane curvature and bending elasticity. Biophys. J. 73, 267-276.

Chernomordik, L. V., and Kozlov, M. M. (2008). Mechanics of membrane fusion. Nat. Struct. Mol. Biol. 15, 675-683.

Churchward, M. A., Rogasevskaia, T., Brandman, D. M., Khosravani, H., Nava, P., Atkinson, J. K., et al. (2008). Specific lipids supply critical negative spontaneous curvature an essential component of native Ca2+-triggered membrane fusion. Biophys. J. 94, 3976-3986. 
Coorssen, J. R., and Rand, R. P. (1990). Effects of cholesterol on the structural transitions induced by diacylglycerol in phosphatidylcholine and phosphatidylethanolamine bilayer systems. Biochem. Cell Biol. 68, 65-69.

Henkel, A. W., Meiri, H., Horstmann, H., Lindau, M., and Almers, W. (2000). Rhythmic opening and closing of vesicles during constitutive exo- and endocytosis in chromaffin cells. EMBO J. 19, 84-93.

Heuser, J., and Reese, T. (1973). Evidence for recycling of synaptic vesicle membrane during transmitter release at the frog neuromuscular junction. J. Cell Biol. 57, 315-344.

Jahn, R., Lang, T., and Südhof, T. (2003). Membrane fusion. Cell 112, 519-533.

Jesenek, D., Perutková, S., Kralj-Iglič, V., Kralj, S., and Iglič, A. (2012). Exocytotic fusion pore stability and topological defects in the membrane with orientational degree of ordering. Cell Calcium 52, 277-282.

Jorgačevski, J., Fosnaric, M., Vardjan, N., Stenovec, M., Potokar, M., Kreft, M., et al. (2010). Fusion pore stability of peptidergic vesicles. Mol. Membr. Biol. 27, 65-80.

Jorgačevski, J., Potokar, M., Grilc, S., Kreft, M., Liu, W., Barclay, J. W., et al. (2011). Munc18-1 tuning of vesicle merger and fusion pore properties. J. Neurosci. 31, 9055-9066.

Jorgačevski, J., Stenovec, M., Kreft, M., Bajic, A., Rituper, B., Vardjan, N., et al. (2008). Hypotonicity and peptide discharge from a single vesicle.
Am. J. Physiol. Cell Physiol. 295, C624-C631.

Kabaso, D., Calejo, A. I., Jorgačevski, J., Kreft, M., Zorec, R., and Iglič, A. (2012). Fusion pore diameter regulation by cations modulating local membrane anisotropy. ScientificWorldJournal 2012:983138. doi: 10.1100/2012/983138

Kozlov, M., and Markin, V. (1983). Possible mechanism of membrane fusion. Biofizika 28, 242-247.

Kreft, M., and Zorec, R. (1997). Cell-attached measurements of attofarad capacitance steps in rat melanotrophs. Pflugers Arch. 434, 212-214.

Lindau, M. (1991). Time-resolved capacitance measurements: monitoring exocytosis in single cells. $Q$. Rev. Biophys. 24, 75-101.

Lindau, M., and Neher, E. (1988). Patch-clamp techniques for timeresolved capacitance measurements in single cells. Pflugers Arch. 411, 137-146.

Lollike, K., Borregaard, N., and Lindau, M. (1995). The exocytotic fusion pore of small granules has a conductance similar to an ion channel. J. Cell Biol. 129, 99-104.

Lollike, K., and Lindau, M. (1999). Membrane capacitance techniques to monitor granule exocytosis in neutrophils. J. Immunol. Methods 232, 111-120.

Malarkey, E. B., and Parpura, V. (2011). Temporal characteristics of vesicular fusion in astrocytes: examination of synaptobrevin 2-laden vesicles at single vesicle resolution. J. Physiol. 589, 4271-4300.

Neher, E., and Marty, A. (1982). Discrete changes of cell membrane capacitance observed under conditions of enhanced secretion in bovine adrenal chromaffin cells. Proc. Natl. Acad. Sci. U.S.A. 79, 6712-6716.

Parpura, V., Basarsky, T. A., Liu, F., Jeftinija, K., Jeftinija, S., and Haydon, P. G. (1994). Glutamate-mediated astrocyteneuron signalling. Nature 369, 744-747.

Parpura, V., and Zorec, R. (2010) Gliotransmission: exocytotic release from astrocytes. Brain Res. Rev. 63, 83-92.

Rituper, B., Flašker, A., Guček, A., Chowdhury, H. H., and Zorec, R. (2012). Cholesterol and regulated exocytosis: a requirement for unitary exocytotic events. Cell Calcium 52, 250-258.

Rosenboom, H., and Lindau, M. (1994). Exo-endocytosis and closing of the fission pore during endocytosis in single pituitary nerve terminals internally perfused with high calcium concentrations. Proc. Natl. Acad. Sci. U.S.A. 91, 5267-5271.

Scepek, S., and Lindau, M. (1993). Focal exocytosis by eosinophilscompound exocytosis and cumulative fusion. EMBO J. 12, 1811-1817.

Schwartz, J., and Wilson, D. (1992). Preparation and characterization of type 1 astrocytes cultured from adult rat cortex, cerebellum, and striatum. Glia 5, 75-80.

Stenovec, M., Kreft, M., Poberaj, I., Betz, W., and Zorec, R. (2004). Slow spontaneous secretion from single large dense-core vesicles monitored in neuroendocrine cells. FASEB J. 18, 1270-1272.
Vardjan, N., Stenovec, M., Jorgačevski, J., Kreft, M., Grilc, S., and Zorec, R. (2009). The fusion pore and vesicle cargo discharge modulation. Ann. N.Y. Acad. Sci. 1152, 135-144.

Vardjan, N., Stenovec, M., Jorgačevski, J., Kreft, M., and Zorec, R. (2007). Subnanometer fusion pores in spontaneous exocytosis of peptidergic vesicles. J. Neurosci. 27, 4737-4746.

White, J. (1992). Membrane fusion. Science 258, 917-924.

Conflict of Interest Statement: The authors declare that the research was conducted in the absence of any commercial or financial relationships that could be construed as a potential conflict of interest.

Received: 24 January 2013; accepted: 14 March 2013; published online: 04 April 2013.

Citation: Kabaso D, Jorgačevski J, Calejo AI, Flašker A, Guček A, Kreft $M$ and Zorec $R$ (2013) Comparison of unitary exocytic events in pituitary lactotrophs and in astrocytes: modeling the discrete open fusion-pore states. Front. Cell. Neurosci. 7:33. doi: 10.3389/fncel. 2013.00033

Copyright (C) 2013 Kabaso, Jorgačevski, Calejo, Flašker, Guček, Kreft and Zorec. This is an open-access article distributed under the terms of the Creative Commons Attribution License, which permits use, distribution and reproduction in other forums, provided the original authors and source are credited and subject to any copyright notices concerning any third-party graphics etc. 